\title{
Early modulation of visual perception by emotional arousal: Evidence from steady-state visual evoked brain potentials
}

\author{
ANDREAS KEIL \\ University of Konstanz, Konstanz, Germany \\ THOMAS GRUBER and MATTHIAS M. MÜLLER \\ University of Liverpool, Liverpool, England \\ STEPHAN MORATTI and MARGARITA STOLAROVA \\ University of Konstanz, Konstanz, Germany \\ and \\ MARGARET M. BRADLEY and PETER J. LANG \\ University of Florida, Gainesville, Florida
}

\begin{abstract}
Allocation of processing resources to emotional picture stimuli was examined using steady-statevisual evoked brain potentials (ssVEPs). Participants viewed a set of 60 colored affective pictures from the International Affective Picture System, presented in a flickering mode at $10 \mathrm{~Hz}$ in order to elicit ssVEPs. Phase and amplitude of the 10-Hz ssVEP were examined for six picture categories: threat and mutilation (unpleasant), families and erotica (pleasant), and household objects and persons (neutral). Self-reported affective arousal and hedonic valence of the picture stimuli were assessed by means of subjective ratings. Viewing affectivelyarousing (unpleasant and pleasant) pictures was associated with enhanced ssVEP amplitude at parieto-occipital recording sites, as compared with neutral stimuli. Phase information suggested increased coactivation of right occipitotemporal and frontotemporal sources during processing of affectively arousing stimuli. These findings are consistent with reentrant modulation of early visual processing by distributed networks including subcortical and neocortical structures according to a stimulus's motivational relevance.
\end{abstract}

Recent theoretical approaches to emotional perception have proposed that the affective/motivational relevance of a visual stimulus may result in changes of sensory processing (Lang, Bradley, \& Cuthbert, 1990). Accordingly, central nervous correlates of affective picture processing have been investigated using a variety of recording techniques and experimental designs (see Cacioppo \& Gardner, 1999, for a review). Several reports have described event-related potentials (ERPs) associated with affective picture viewing (Cuthbert, Schupp, Bradley, McManis, \& Lang, 1998; Ito, Larsen, Smith, \& Cacioppo, 1998; Mini, Palomba, Angrilli, \& Bravi, 1996; Palomba, Angrilli, \& Mini, 1997). These ERP studies have consistently demonstrated a sustained late positive wave ( $>300$-msec latency) in response to emotional stimuli, which was attenuated when subjects viewed calm pictures. Recent work using dense-array EEG and distributed source modeling have shown that these late ERP effects appear to be generated

This work was supported by the Deutsche Forschungsgemeinschaft. The authors thank Ursula Lommen for help in data acquisition. Correspondence concerning this article should be addressed to A. Keil, Department of Psychology, University of Konstanz, Box D23, D-78457 Konstanz, Germany (e-mail: andreas.keil@uni-konstanz.de). in visual areas of the occipital and temporal cortex, as well as in right-hemispheric parietal areas (Keil et al., 2002).

Reentrant modulation of the visual cortex has been discussed as a mechanism that enhances processing when a stimulus is important. In addition to late ERP effects, earlier ERP differences in time windows between 100 and $300 \mathrm{msec}$ after stimulus onset have been reported, supporting the view that the amplitude of sensory processing at relatively early stages is modulated as a function of stimulus significance (Junghöfer, Bradley, Elbert, \& Lang, 2001). For example, using a hemifield design with affective pictures from the International Affective Picture System (IAPS), Keil and collaborators (Keil, Müller, et al., 2001) reported differences of the N1 amplitude for arousing, as compared with neutral stimuli. In analogy to findings in the field of selective attention (Hillyard \& Anllo-Vento, 1998), a sensory gain mechanism has been hypothesized to amplify sensory processing according to the importance of the stimulus for the organism. This would be consistent with reaction time (Hartikainen, Ogawa, \& Knight, 2000; Öhman, Flykt, \& Esteves, 2001) and memory performance (Bradley, Greenwald, Petry, \& Lang, 1992) data and has been referred to as motivated attention (Lang, Bradley, \& Cuthbert, 1997b). 
In line with the idea of sensory modulation by the brain's motivational system, work in which metabolic and blood flow changes during the viewing of emotional scenes or pictures have been investigated has suggested differential functional activation of a variety of cortical and subcortical structures, depending on the emotional content of picture stimuli. There has been, however, considerable variability across studies regarding kind and degree of involvement of these structures (Davidson, Pizzagalli, Nitschke, \& Putnam, 2002). Moreover, the relation of these data to different aspects of affective processing, such as evaluative judgment, action preparation, or modulation of perception, has made interpretation of imaging results difficult.

In a study using functional magnetic resonance imaging (fMRI), Lang and collaborators (Lang et al., 1998) examined the hypothesis that the intensity of neuronal activity in the visual cortex is enhanced with increasing motivational significance of the visual stimulus. They found that the functional activation in visual areas of the occipital cortex varied as a function of affective arousal. Significant enhancement of the blood oxygen level dependent (BOLD) response was related to viewing both pleasant and unpleasant pictures, as compared with neutral stimuli. These arousal-related differences were more pronounced in the right hemisphere. The authors suggested that reentrant afferents to visual cortices modulate processing of a given stimulus according to its motivational significance. Given the low temporal resolution of the BOLD response and its relation to metabolic processes, rather than to neuronal functioning, it is desirable to use measures that complement the information obtained by hemodynamic imaging techniques. In particular, examining the temporal relations between brain areas during the processing of emotional stimuli differing in emotional arousal (intensity) or valence may be useful for understanding the nature of modulatory input to the visual system. For instance, Junghöfer and collaborators have recorded ERPs in a rapid serial picture presentation paradigm to investigate differences in visual processing related to emotional arousal (Junghöfer et al., 2001). They reported enhanced negativity at posterior recordings sites in time ranges around $200 \mathrm{msec}$ after onset of arousing, as compared with neutral, pictures, supporting the idea of affect modulation at relatively early stages of visual processing.

\section{THE RESEARCH PROBLEM}

In the present research, emotional picture processing was assessed using the steady-state visual evoked potential (ssVEP). This method had been used previously to investigate modulatory processes in the field of visual selective attention. The ssVEP is a continuous brain response elicited by a repetitive visual stimulus (see Figure $1 \mathrm{~A}$ ) that is periodically modulated in intensity at a fixed rate of $6-8 \mathrm{~Hz}$ or greater. Using standard psychophysiological techniques, these signals can be recorded at the scalp as an oscillatory waveform that has the same fundamental frequency as the driving stimulus, often including higher harmonics (Regan, 1989; see Figure 1B). The topography of this oscillatory response shows a typical time course, suggesting that its generators are located primarily in visual areas. This is illustrated in Figure $1 \mathrm{C}$. Depending on the driving frequency, maximum amplitudes are seen at parieto-occipital sites but also show ventrolateral amplitude enhancement (Figure 2A). Accordingly, localization studies have converged to show that the signal recorded at the surface of the scalp is likely to be generated in parieto-occipital cortices, with a sensitivity of the sources to the driving frequency (Müller, Teder, \& Hillyard, 1997).

In terms of data quality, ssVEP paradigms allow the acquisition of a high number of trials in a short period of time, thus having excellent signal-to-noise ratio (Mast \& Victor, 1991). Therefore, small differences in amplitude or timing can be identified. While amplitude measures may indicate recording sites with enhanced involvement in the oscillatory activity, phase information can be used to complement this information by a brain dynamic aspect (Burkitt, Silberstein, Cadusch, \& Wood, 2000; see Figures $2 \mathrm{~B}$ and 2C). For example, topography of phase information can help to identify underlying brain-electric current sources that are associated with a reversal of the phase of the signal, such as changing from a positivegoing to a negative-going waveform. Furthermore, phase differences between experimental conditions may indicate task-dependent brain response latency changes, as well as changes in source location or extension. Given these properties, steady-state potentials appear to be especially suitable for monitoring processes related to fluctuations in transient states of the central nervous system, such as attention or emotional arousal. An additional advantage can be seen in the fact that the relevant parameters (i.e., amplitude and phase of the ssVEP) can be easily extracted by means of frequency domain analyses, such as fast Fourier transform (FFT), and submitted to further analyses (Müller, Picton, et al., 1998; Picton, Vajsar, Rodriguez, \& Campbell, 1987).

Steady-state potentials in the auditory or visual modality have been shown to be sensitive for tonic subjective states, such as anesthesia (Plourde \& Picton, 1990), sleep (Picton et al., 1987), or vigilance (Silberstein et al., 1990). In addition, the amplitudes of ssVEPs are modulated as a function of phasic changes in the individual, such as cognitive performance (Silberstein, Ciorciari, \& Pipingas, 1995) or visual spatial selective attention (Morgan, Hansen, \& Hillyard, 1996). Thus, the amplitude of the ssVEP recorded at sites contralateral to the attended location in a spatial attention paradigm was enhanced, as compared with control conditions (e.g., Müller, Picton, et al., 1998). These findings were not related to specific frequencies but were observed for modulation rates ranging from 9 to $28 \mathrm{~Hz}$.

Given the relationship between affective/motivational aspects of a stimulus and processes of motivated atten- 
$10-\mathrm{HZ}$

stimulation

A
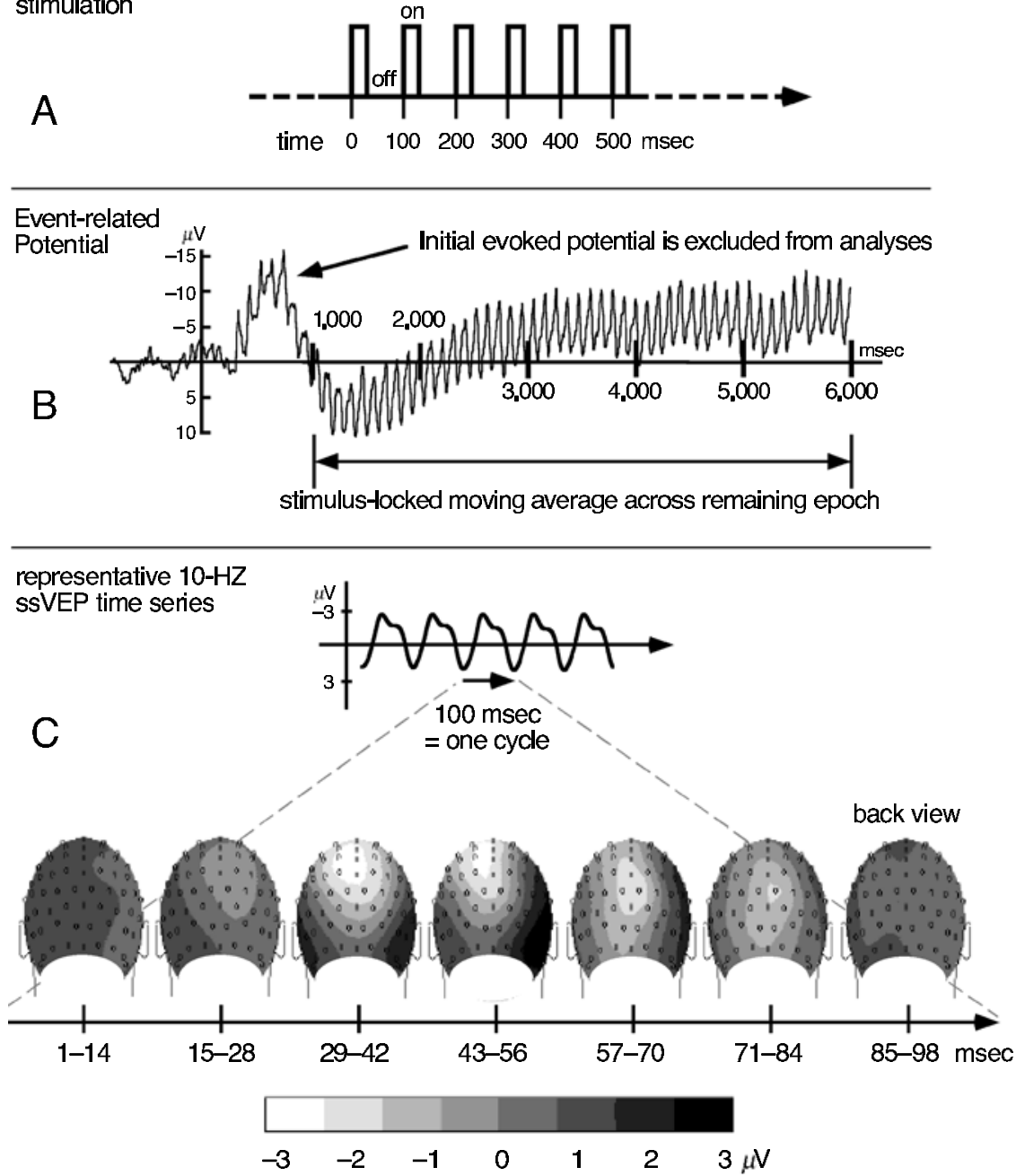

Figure 1. Recording and data reduction of steady-state visual evoked potentials (ssVEPs): time course and topography for an individual participant. A neutral stimulus was presented flickering at a rate of $10 \mathrm{~Hz}$. (A) This elicited a visual evoked potential, followed by a steadystate signal, as is visible after averaging across trials. (B) The steady-state signal is further averaged across time using a moving average procedure, resulting in a representative time series at each sensor. (C) Topographical changes of voltage during one $10-\mathrm{Hz}$ ssVEP cycle (= $100 \mathrm{msec}$ ) are shown at the bottom.

tion, we expected ssVEP amplitude and phase alterations to be associated with variations on the dimension of affective arousal. In the present study, we focused on amplitude and timing of neural mass activity, rather than aiming at the identification of their neuroanatomical correlates. For stimulation, we used color pictures from the IAPS (Lang, Bradley, \& Cuthbert, 1997a). The IAPS includes standard ratings for each picture as to its hedonic valence, emotional arousal, and dominance, obtained from a normative sample of subjects. It is based on a dimensional approach of emotion, allowing for systematic variations of stimuli along the dimensions of emotional valence and arousal (Lang, 1995). In analogy to work in the field of selective attention and on the basis of the imaging results described above, we hypothesized that the ssVEP amplitude would be enhanced when affectively arousing pictures were viewed. The locations of these differences were expected in the occipital and parietal cortex. In addition, processing stimuli differing in affective valence was assumed to be associated with effects on the latency and source configuration of the brain response, reflected in the phase of the ssVEP.

\section{METHOD}

\section{Subjects}

Twenty-two paid volunteers ( 13 females and 9 males) gave written informed consent to participate in the study. Their mean age was 26.4 years. They had normal or corrected-to-normal vision and no 


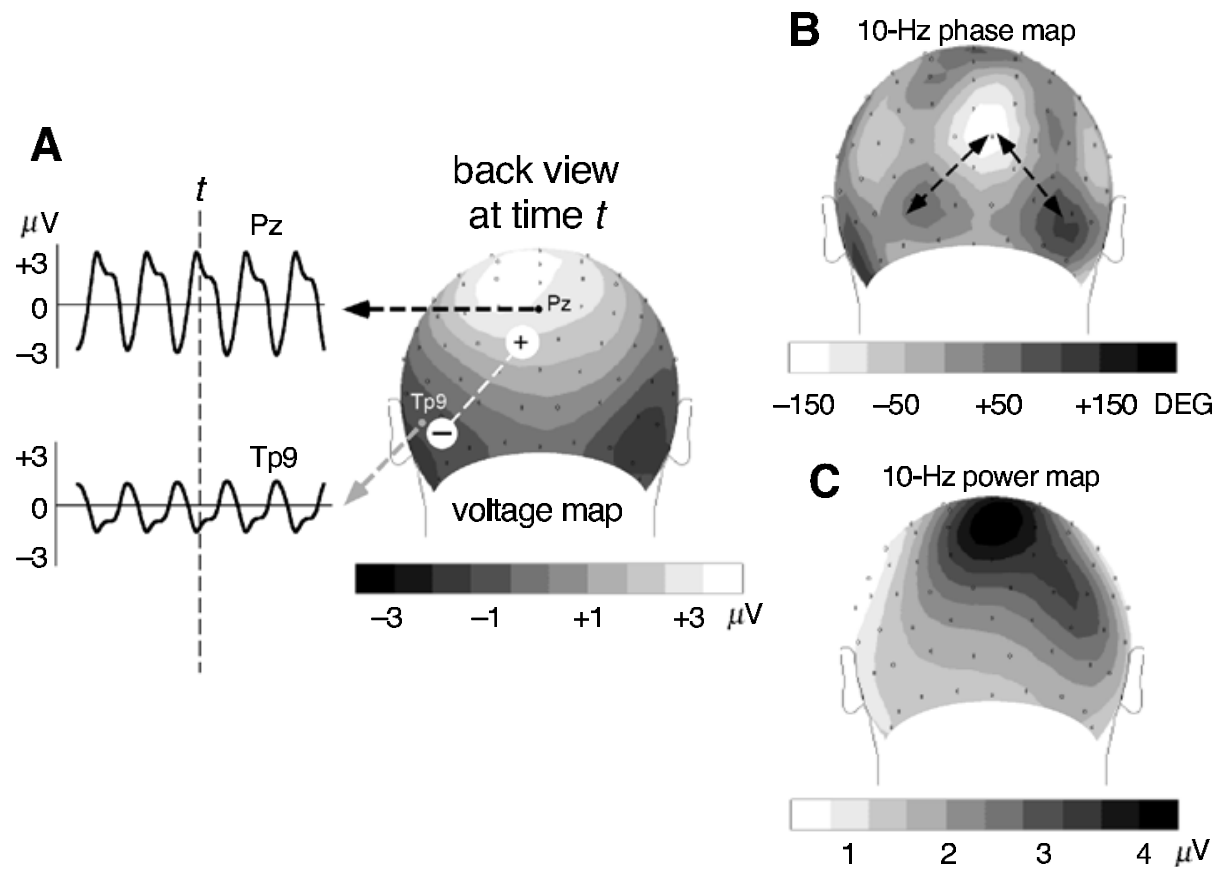

Figure 2. Relationship between time domain signal and frequency domain phase and amplitude/power in 10-Hz steady-state visual evoked potentials. Voltage reversals and latency changes of the time series at different sensors (A) are represented as phase changes in the phase map (B). The spectral power map $(C)$ is sensitive for the amount of energy present in the $10-\mathrm{Hz}$ frequency band at each EEG sensor, irrespective of phase (latency) differences.

family history of photic epilepsy. Data from 3 subjects were discarded because of lack of an ssVEP signal (see the Results section). Thus, 19 participants (11 females and 8 males) were included in the analyses reported below.

\section{Stimuli}

Sixty colored pictures from the IAPS (Lang et al., 1997a) were used as stimuli. This collection of pictures has normative ratings with respect to the dimensions of affective valence (i.e., pleasant vs. unpleasant) and arousal (calm vs. aroused). Affective valence and arousal were manipulated by presenting 20 pictures depicting erotic couples or happy families (pleasant), 20 pictures depicting emotionally neutral persons or household objects (neutral), and 20 pictures depicting mutilated bodies or threat scenes (unpleasant). ${ }^{1} \mathrm{Lu}$ minance, contrast, and color spectrum of IAPS pictures were determined using a luminance meter and digital image processing software (MATLAB 5.2). Adobe Photoshop 3.0 was used to adjust mean luminance, as well as mean red, green, and blue values, so that there were no systematic differences between the six picture categories. The mean luminance of the pictures, as measured in the experimental chamber, was kept between 12.5 and $22.5 \mathrm{~cd} / \mathrm{m}^{2}$. Likewise, the luminance of picture centers was kept between 15.2 and $26.0 \mathrm{~cd} / \mathrm{m}^{2}$.

The pictures were presented in a pseudorandom order on a 19-in. monitor with a vertical refresh rate of $70 \mathrm{~Hz}$, subtending a visual angle of $7^{\circ}$ both horizontally and vertically. A fixation point was marked in the center of the screen and was present throughout the experiment. During one trial, a picture was presented in a flickering mode at a rate of $10 \mathrm{~Hz}$ for a period of $6,000 \mathrm{msec}$, thus containing $60 \mathrm{on} /$ off cycles, the picture being shown for $43 \mathrm{msec}$, followed by a 57-msec black screen during each cycle. A $10-\mathrm{Hz}$ stimulation was used because it is related to high ssVEP amplitudes in human sub- jects and thus allows a favorable signal-to-noise ratio, as compared with faster driving frequencies. The intertrial interval varied randomly between 8 and $12 \mathrm{sec}$.

\section{Procedure and Design}

Upon arriving at the laboratory, the subjects completed an informed consent form and were introduced to the stimulus material by viewing examples of IAPS pictures that were not part of the experimental set. After application of the electrode net, they were seated in a comfortable chair, and the experimental session started with the presentation of 60 flickering stimuli in a pseudorandomized order. The subjects were instructed to view the pictures attentively and to maintain gaze on the central fixation spot. They were also asked to avoid blinks and eye movements during presentation of the stimuli. Randomization was constrained in that a straight sequence of 3 pictures from the same affective category did not occur. After this first block, the subjects were offered a brief pause, and a second block of the same 60 pictures in changed order was run. Subsequently, the sensor net was removed, and the subjects viewed the pictures a third time. In this last block, they were asked to rate each picture as to its valence (pleasant vs. unpleasant) and arousal (calm vs. arousing), using the Self-Assessment Manikin (SAM) self-report scale (Lang, 1980).

\section{EEG Recordings and Data Analysis}

EEG was recorded from 129 electrodes, using an Electrical Geodesics system. Data were sampled at a rate of $250 \mathrm{~Hz}$, constrained by on-line bandpass filtering between 0.1 and $100 \mathrm{~Hz}$. Eye movements and blinks were controlled by visually inspecting the vertical and horizontal electrooculogram (EOG) as computed from a subset of the electrodes that were part of the electrode net. Artifact rejection was performed off line, following the procedure proposed 
by Junghöfer and co-workers (Junghöfer, Elbert, Tucker, \& Rockstroh, 2000), which provides a four-step approach to artifact rejection in multichannel EEG data. It (1) detects individual recording channel artifacts, using the recording reference (i.e., Cz), (2) converts data to average reference and detects global artifacts, (3) replaces those sensors that are contaminated with artifacts throughout with statistically weighted spherical spline interpolation from the full channel set, and (4) computes the variance of the signal across trials to document the stability of the averaged waveform. The maximum number of approximated channels was set to 20 , resulting in an overall rejection rate of $19 \%$ of the trials. Epochs of 200 -msec prestimulus and 6,000-msec poststimulus onset were obtained. Artifact-free epochs were averaged separately for the six affective categories, and a 200-msec prestimulus data segment was subtracted as baseline. To eliminate the effects of initial ERPs to stimulus train onset, a time period of 1,000-6,000 msec after train onset was used for all the subsequent analyses.

Assessment of ssVEP presence. For evaluating the presence of an ssVEP different from noise in each subject, the 1,000- to $6,000-$ msec poststimulus part of these averaged potentials was divided into five temporally equidistant, nonoverlapping sections of 1,000 msec length, each of which served as an independent sample for estimating the $10-\mathrm{Hz}$ Fourier components. These were demeaned and de-trended using linear regression. Subsequently, a $500-\mathrm{msec}$ window containing five cycles of the $10-\mathrm{Hz}$ flickering stimuli was shifted in steps of $100 \mathrm{msec}$ (i.e., 25 data points), and the potential within the shifting windows in the time domain was further averaged (see Figure 1B). The resulting ssVEP data epochs of $500 \mathrm{msec}$ (see Figure 3) were transformed into the frequency domain, using FFT on 128 data points, whereby 3 data points were padded with zeros. Thus, Fourier coefficients reflecting the frequency of interest (i.e., $10 \mathrm{~Hz}$ ) were obtained for three affective categories and five data segments, respectively. Consequently, 15 independent estimates for the $10-\mathrm{Hz}$ Fourier coefficients were submitted to the circular T-square algorithm proposed by Victor and Mast (Mast \& Victor, 1991; Victor \& Mast, 1991), which exploits the fact that real and imagery parts of the Fourier components are independent. It calculates a test statistic $T_{\text {circ }}^{2}$ on the basis of the variance estimates for the sample and an assumed population mean. For assessment of signal presence, the value for the population mean was set to zero, and the $T_{\text {circ }}^{2}$ statistic was computed for all subjects and EEG channels. We required that significant $(p<.05) T_{\text {circ }}^{2}$ values were obtained at least at 10 out of 16 electrode locations around site Poz of the international 10-20 system. This group of sensors (see Figure 3) also served for data reduction, as will be described below.

Assessm ent of ssVEP condition differences. For evaluating differences in ssVEP phase and amplitude between affective picture categories, $10-\mathrm{Hz}$ Fourier components were obtained for a 500 -msec data segment resulting from the moving average across the poststimulus onset part of the epoch following the initial ERPthat is, $1,000-6,000 \mathrm{msec}$. This was done in order to further increase the signal-to-noise ratio for the stimulus-locked response. Differences of the ssVEP between affective categories were then evaluated for each recording channel by submitting each subject's Fourier coefficients to pairwise comparisons using the $T_{\text {circ }}^{2}$ statistic for assessment of differences between two means, resulting in the across-subjects comparisons neutral versus pleasant, neutral versus unpleasant, and pleasant versus unpleasant. Because of the problems associated with multiple significance testing, $P$ value adjustments were made according to the permutation method proposed by Blair and Karniski (Blair \& Karniski, 1993; Karniski, Blair, \& Snider, 1994). For the present data set, this procedure re-

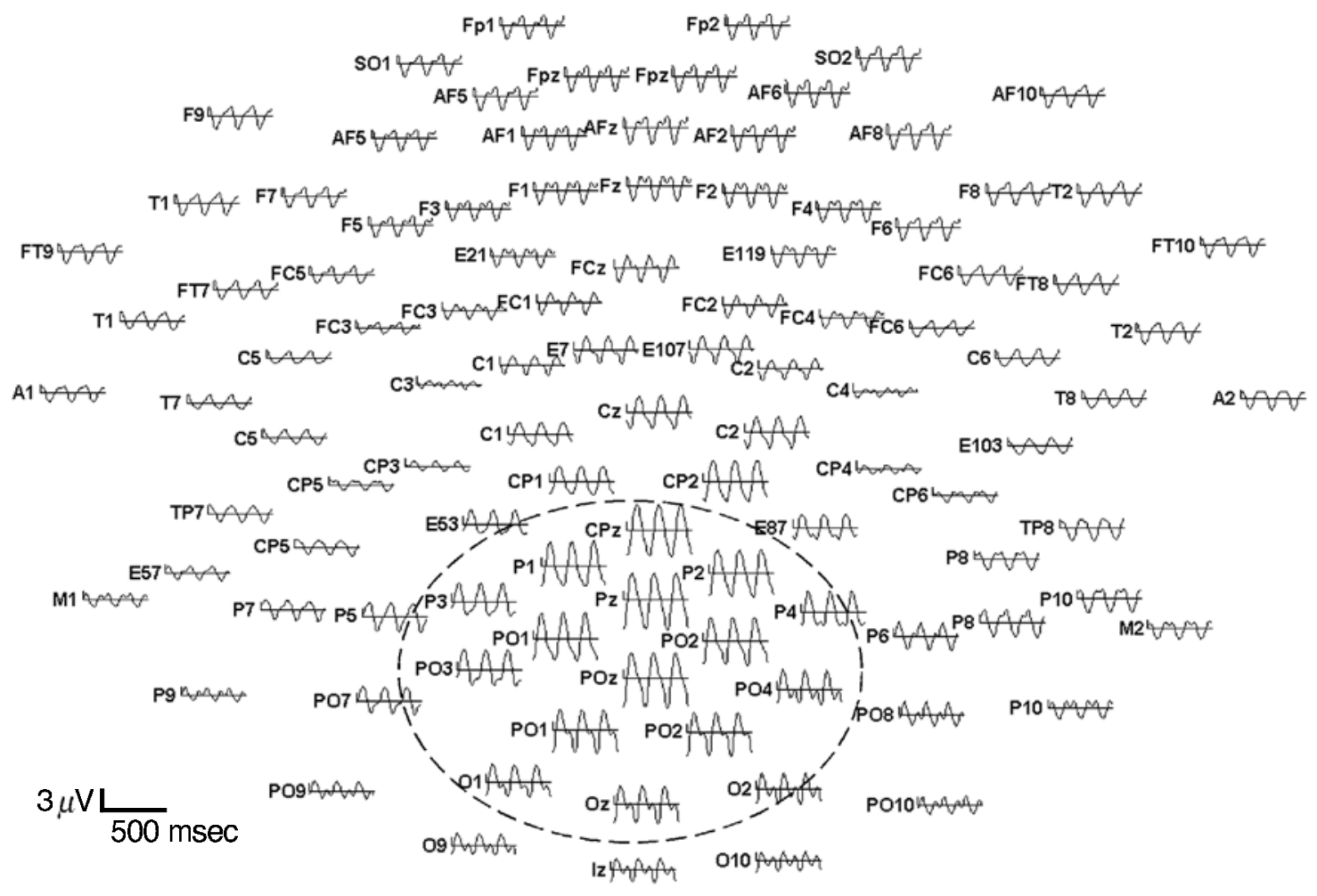

Figure 3. Grand mean $(n=19)$ steady-state visual evoked potentials at a subset of electrodes, roughly corresponding to sites of the International 10-20 System, for unpleasant picture content. The electrode group used for computing posterior regional means is encircled. Note: positive is up. 
sulted in critical $F$ values of $11.3(p<.05), 16.2(p<.01)$, and 34.6 $(p<.001)$, respectively.

As a second approach to testing for differences at posterior recording sites, an analysis of variance (ANOVA) on a posterior regional mean was used. To this end, amplitude and phase were calculated from the $10-\mathrm{Hz}$ Fourier components for each affective category and electrode. A regional amplitude mean for 16 parieto-occipital electrodes between sites $\mathrm{CPz}$ and $\mathrm{Oz}$ of the international 10-20 system (see Figure 3 ) was determined and was analyzed by means of a twostep procedure. First, we conducted ANOVAs having the withinsubjects factor of category (pleasant, neutral, or unpleasant) and the between between-subjects factor of gender. Trend analyses (planned comparisons) were used for examining the expected relationship between ssVEP amplitudes and picture arousal across categories. The same ANOVA model was used for self-reported arousal and valence. In a second step, significant effects of category were followed by ANOVAs for specific contents in each category, resulting in a within-subjects factor of content (two contents per category) and the between-subjects factor gender. For all ANOVAs, GreenhouseGeisser correction was applied when necessary (Greenhouse \& Geisser, 1959). In the following, corrected $p$ values are reported, where appropriate.

\section{RESULTS}

\section{Self-Report Data}

As was expected, the ANOVAs on the the subjects' SAM ratings showed that the picture categories differed significantly as to their valence $[F(2,34)=386.0, p<$ $.001]$. Trend analyses revealed that the valence ratings of all the affective categories differed linearly from each other $[F(1,18)=387.1, p<.001]$. Gender interacted with picture category $[F(2,34)=10.5, p<.01]$, with women showing higher pleasure ratings and aversion ratings than did men for pleasant and unpleasant pictures, respectively. Regarding subcategories, content differences were observed for pleasant and unpleasant categories. Family content was associated with higher pleasure ratings than was erotica $[F(1,17)=4.5, p<.05]$, which was due to women's showing enhanced ratings for family pic- tures, as indexed by the interaction of content and gender $[F(1,17)=5.1, p<.05]$. With respect to the unpleasant category, mutilation was rated as being more unpleasant than threat $[F(1,17)=31.7, p<.001]$, and women had lower pleasure scores for this category across contents $[F(1,17)=8.5, p<.01]$.

Arousal ratings showed a significant effect of category as well $[F(2,34)=80.5, p<.001]$, displaying a quadratic trend with increased ratings for pleasant and unpleasant, as compared with neutral, pictures $[F(1,18)=157.7, p<$ .001 ; see Figure 4, left]. All the categories showed differences in self-rated arousal for specific contents. Erotica were rated as being more arousing than families $[F(1,17)=$ $18.4, p<.001]$, and neutral persons had higher arousal ratings than did household objects $[F(1,17)=26.4, p<$ $.001]$. In terms of unpleasant contents, mutilations were rated as being more arousing than threat $[F(1,17)=9.9$, $p<.01]$, and an effect of gender $[F(1,17)=5.9, p<.01]$ emerged, showing higher arousal ratings in women than in men for unpleasant contents specifically.

Thus, pictures were experienced according to their affective categories. Similar effects have been reported in a variety of studies using IAPS pictures and SAM ratings as a dependent variable (e.g., Bradley, Codispoti, Sabatinelli, \& Lang, 2001; Bradley \& Lang, 1994; Hamm, Greenwald, Bradley, \& Lang, 1993).

\section{sSVEPs}

Presence of ssVEP signals. The signals from 3 subjects showed no significant $T_{\text {circ }}^{2}$ statistics for at least 10 channels between sites corresponding to $\mathrm{Pz}$ and $\mathrm{Oz}$ from the international 10-20 system, where the ssVEP signal was most pronounced. Thus, data from these subjects were discarded because of this criterion. Accordingly, data from the 19 subjects ( 11 females and 8 males) with significant ssVEP responses were submitted to further analyses.
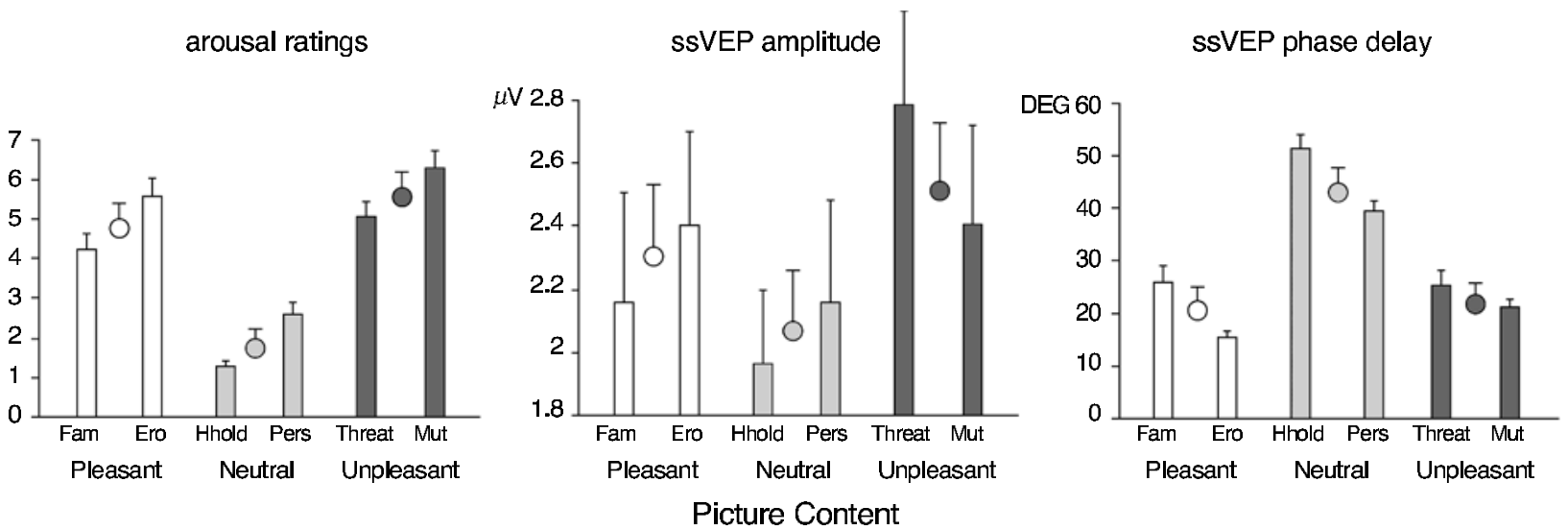

Figure 4. Left: Results of subjective ratings using the Self-Assessment Manikin scale. Means across participants $(n=19)$ and pictures for six contents are shown. Bars indicate standard errors. Middle: Regional posterior means of 10-Hz amplitude for six picture contents. Bars indicate standard errors. Right: Regional posterior means of 10-Hz phase. As a measure of variability across participants, bars indicate circular variance, which is bounded between 0 and 1 , multiplied by a factor of 10 to be visible on this scale. Circles between bars represent mean values across specific contents for pleasant, neutral, and unpleasant categories. 
sSVEP amplitude and phase. The ANOVA on posterior regional means revealed that the amplitude of the $10-\mathrm{Hz}$ ss VEP was enhanced during viewing pleasant (grand mean regional amplitude at posterior sites $=$ $2.34 \mu \mathrm{V})$ and unpleasant $(2.51 \mu \mathrm{V})$, as compared with neutral $(2.06 \mu \mathrm{V})$, pictures $[F(2,34)=5.1, p<.01$; see Figure 4, middle]. This was also visible in the time domain, as represented by a posterior regional mean (see Figure 5). Thus, the amplitude data showed a significant quadratic trend, reflecting modulation by emotional arousal $[F(1,18)=5.9, p<.05]$. In terms of specific content, threat pictures were associated with higher amplitudes than were mutilation pictures $[F(1,17)=10.1 p<$ $.01]$ across sexes. In contrast to subjective ratings, no significant main effect or interaction involving specific picture content or the subjects' gender was observed in the pleasant and neutral picture categories.

As depicted in the grand mean spline-interpolated amplitude maps shown in Figure 6, the topographical distribution of the ssVEP amplitude was similar across affective categories. The maximum of the grand mean potential distribution was seen at electrodes corresponding to $\mathrm{Pz}$ across affective categories. Around this site, the voltage distribution showed a right-hemispheric preponderance that was most pronounced for neutral pictures.

With respect to ssVEP phase, differences between affective picture categories were most pronounced at posterior and right frontotemporal sites. At posterior sites, where the ssVEP amplitude was strongest, we found a modulation of phase delay as a function of emotional arousal (see Figure 4, right). Relative to $10 \mathrm{~Hz}$ sine, neutral pictures exhibited a bigger phase delay $\left(43^{\circ}\right)$ than did pleasant $\left(19^{\circ}\right)$ and unpleasant $\left(21^{\circ}\right)$ pictures. These regions of amplitude and phase differences between picture categories were confirmed by means of pairwise comparisons using the circular T-square algorithm (see Figure 7). $F$ values were greatest at (1) parieto-occipital recording sites, where the ssVEP amplitude was largest across conditions while consistent phase differences were seen, and (2) frontotemporal recording sites, where phase differences were most pronounced. Using the circular T-square algorithm, we found strong differences between unpleasant and neutral pictures as well as between pleasant and neutral pictures $[F \mathrm{~s}(2,34)>34.6, p<$ $.001]$ at central parieto-occipital electrodes, whereas there were smaller differences between pleasant and unpleasant pictures at those sites $\left[F_{\mathrm{S}}(2,34)>11.3, p<.05\right]$.

Frontotemporal recording sites also showed discrimination of phase between arousing and calm pictures. These sites, however, displayed enhanced phase delay for arousing, as compared with neutral, pictures, with a maximum delay of $22^{\circ}$ for neutral pictures, $79^{\circ}$ for pleasant pictures, and $73^{\circ}$ for unpleasant pictures at the sites showing significant frontotemporal differences, as is depicted in Figure 7.

\section{DISCUSSION}

The aim of the present study was to examine electrocortical correlates of early visual affective stimulus processing, using steady-state brain potentials. We found that the ssVEP amplitude associated with viewing of arousing (pleasant and unpleasant) pictures was enhanced, as compared with neutral pictures. Unpleasant pictures were rated as more arousing than pleasant stimuli in the present study and showed slightly higher brain response amplitudes. Thus, differences in ssVEP amplitudes reflected category differences regarding the subjective ratings on the arousal dimension. This is consistent with the view adopted here-namely, the view that

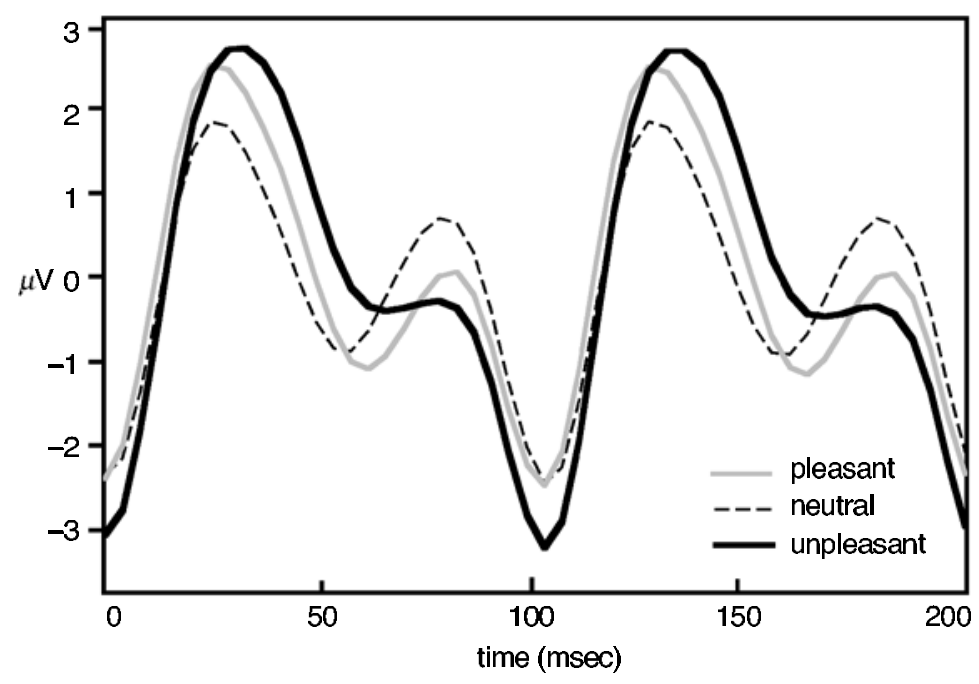

Figure 5. Grand average $(n=19)$ voltage time series for two cycles of the steady-state visual evoked potential at a regional mean of posterior sites around site Poz, shown for pleasant, neutral, and unpleasant picture contents. 


\section{$10-\mathrm{Hz}$ spectral power back view}
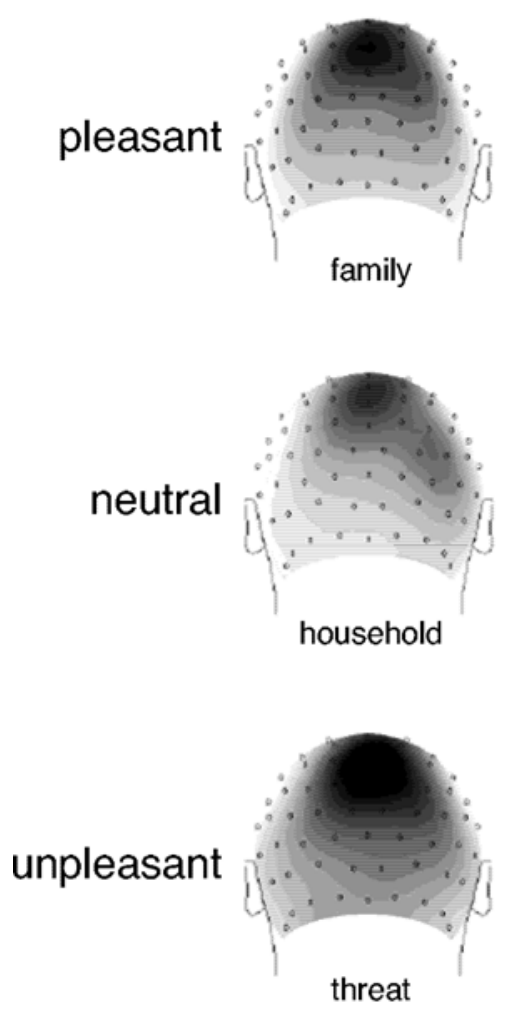
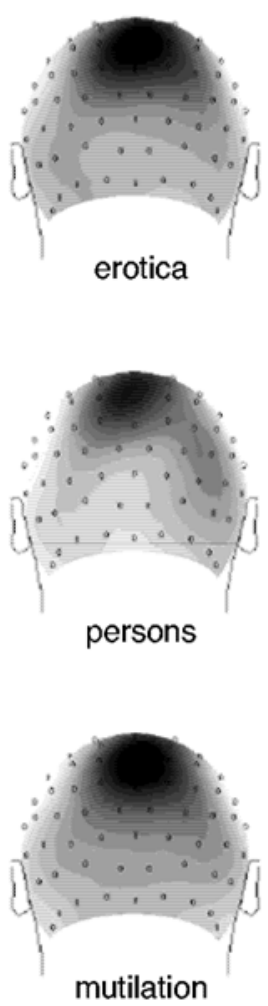

mutilation

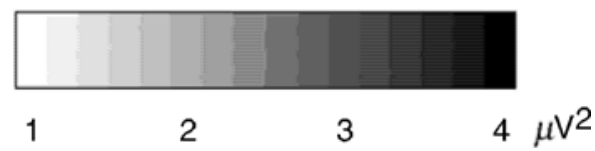

Figure 6. Grand mean $(n=19)$ spline-interpolated topographical distribution of $10-\mathrm{Hz}$ spectral power for six specific contents. A back view is shown.

visual selective attention may be allocated to external stimuli according to their respective motivational or affective significance (Lang et al., 1997b). Paralleling findings in the field of selective attention (Hillyard \& Anllo-Vento, 1998), this may be regarded as an amplification mechanism that serves to enhance sensory processing of relevant information.

Similar interpretations have been suggested for modulations of other electrocortical measures of affective picture processing, such as late components of the ERP (e.g., Schupp et al., 2000), the selection negativity (Schupp, Junghöfer, Weike, \& Hamm, in press), the visual N1 (Keil, Müller, et al., 2001), and the visual N1/P2 component in a rapid serial visual presentation paradigm (Junghöfer et al., 2001). The authors of the latter study suggested that a potential mechanism for early selection of arousing information from a temporal stream might reflect an emotional component of a short-term working memory process. In line with these conjectures, stimulus significance has been shown to enhance visual identification performance in a rapid stream with behavioral paradigms such as the attentional blink (Anderson \& Phelps, 2001), which is assumed to be related to postperceptual processes. An alternative explanation may focus on earlier stages of visual processing. Animal and human work indicates that the functional neuroarchitecture of the adult visual cortex is subject to experience-related changes (Gilbert, 1998) and reentrant modulation from higher visual areas and deep structures, such as the amygdala or the anterior cingulate (Damasio, 1998; Davis, 1998). Accordingly, visual neuronal networks representing affectively relevant information may have acquired stronger connectivity and, possibly, are more widespread than assemblies representing less arousing information 


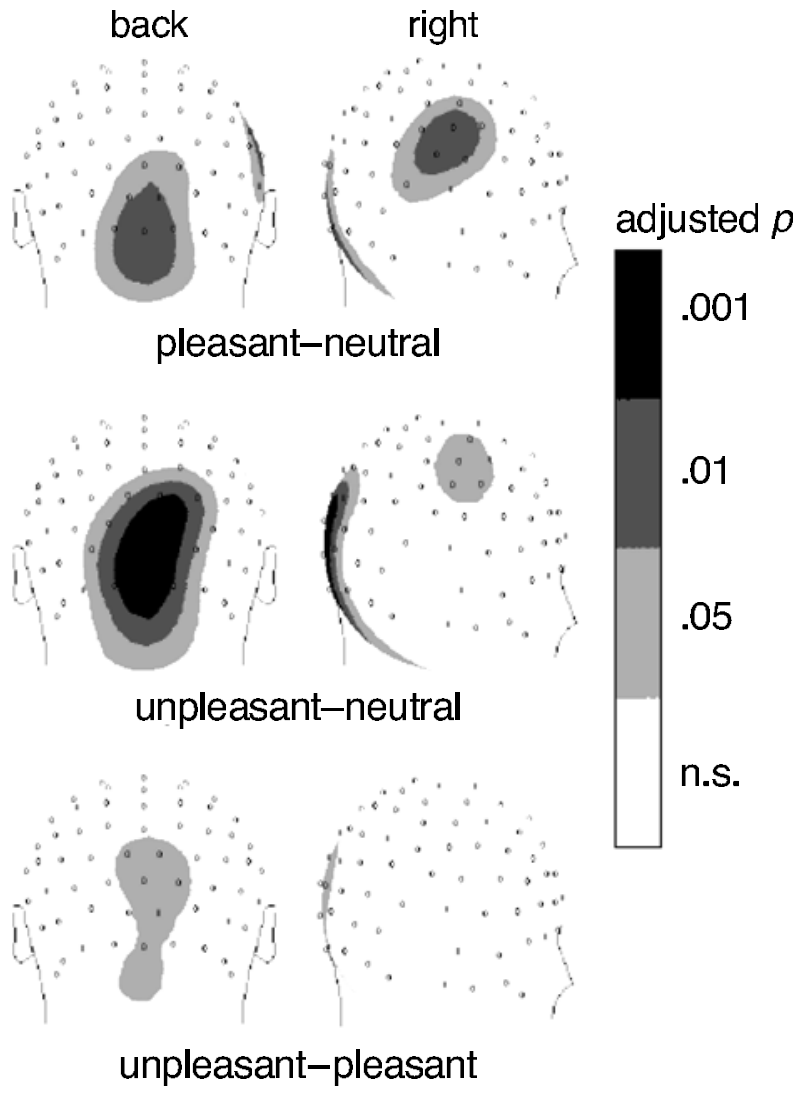

Figure 7. Topographical distribution of significant (corrected $p<.05, p<.01$, and $p<.001$, respectively) differences between pleasant, neutral, and unpleasant picture content as indicated by the circular T-square statistic, computed for each sensor.

(Keil, Gruber, \& Müller, 2001). Thus, the visual response amplitude to arousing stimuli might be enhanced, and timing might be accelerated. This would hold especially in cases in which the emotional content of repeatedly presented stimuli is constant and reentrant modulation can be in effect over a longer period of time, as is the case in ssVEP paradigms. Using IAPS pictures together with ssVEPs elicited by $13-\mathrm{Hz}$ peripheral flicker, Kemp and collaborators (Kemp, Gray, Eide, Silberstein, \& Nathan, 2002) found transient reduction of latency at frontal sites when low-arousal pleasant and unpleasant IAPS pictures were viewed, as compared with neutral pictures. Amplitude of ssVEP background flicker was reduced during perception of unpleasant stimuli, which might be interpreted as desynchronization or activation in frontal networks. Although these findings were obtained for a situation in which ssVEPs were elicited by diffuse peripheral stimulation rather than by the stimulus itself, they are concordant with our report of posterotemporal phase acceleration and amplitude enhancement for the processing of arousing stimuli.

Regarding methodological constraints, the issue of differential eye movements must be considered. As a consequence of motivated attention, increased exploration of picture content would be expected for arousing, as compared with calm, pictures. In our study, we used a central fixation point and monitored eye movement by means of the EOG. In addition, we rejected trials showing outlying scalp distribution reflecting ocular movement, using the SCADS approach described in the Method section. The number of rejected trials did not differ between conditions. Small eye movements might not be detected by means of EOG measures, however. Thus, work using eye-tracking techniques is needed to clarify whether motivated attention to emotionally arousing information is related to involuntary eye movements under fixation instructions.

Gender differences observed in subjective ratings were not reflected in ssVEP measures. As with ratings in our study, previous work has demonstrated enhanced correlation between aversive valence and affective arousal in women, whereas men displayed higher coupling between pleasantness and emotional arousal (Bradley et al., 2001). These differences have also been observed in measures of brain metabolism (Lang et al., 1998). Ratings are affected by a variety of higher order cognitive and behavioral processes, however, which may not interact with initial visual perception as measured by ssVEPs. Furthermore, gender differences were not the focus of the present study, in which only 8 men participated. Work using multivariate approaches and bigger samples are needed to examine issues of gender differences in initial affective perception.

Analysis of ssVEPs contributes to the ERP findings mentioned above by adding information as to the response timing and stimulus-locked amplitude, as provided by frequency domain analyses. Most important, ssVEPs can be evaluated using time frequency analysis, thus allowing one to detect changes and fluctuations occurring during the time course of one stimulus train (Silberstein, Harris, Nield, \& Pipingas, 2000). Although enhancement of ssVEP amplitude in attentional paradigms has been shown at a variety of driving frequencies ranging from 10 to $28 \mathrm{~Hz}$ (e.g., Müller, Teder-Salejarvi, \& Hillyard, 1998), it is unclear whether modulation related to emotional arousal will be present at frequencies other than $10 \mathrm{~Hz}$. Steady-state responses to stimulation at higher frequencies tend to activate lower level regions of the visual cortex (Müller et al., 1997). Thus, replication of our results with a faster rate of presentation is desirable. Future work may also exploit the compatibility of ssVEP designs with fMRI measurement techniques. In the present study, posterior ssVEP phase differences suggested that timing of the brain responses differed as a function of picture arousal. These findings were confirmed by topographical analyses using the circular T-square statistic at each recording site. Whereas amplitude differences were most pronounced at posterior sites, the effects of picture content on the phase of the ssVEP were greatest at frontotemporal recording sites. In addition, phase differences at posterior sites showed a rela- 
tionship with arousal ratings and ss VEP amplitude. This pattern of effects suggests differential coactivation of frontotemporal structures for different contents, which may modulate the ssVEP signal at several stages of visual processing. For instance, temporal cortical areas that are part of object identification networks have been shown to provide reentrant modulatory input into posterior areas of the visual cortex (Amaral, Price, Pitkänen, $\&$ Carmichael, 1992). Caution is warranted, however, as to the interpretation of these changes as accelerated processing in visual areas and delayed processing in more anterior regions. As Silberstein and associates (Silberstein, Nunez, Pipingas, Harris, \& Danieli, 2001) have pointed out, changes of phase may reflect differences in transmission time, axonal length, and the number of synapses involved in the process. In addition, the amount of coupling and synchronicity within the neuronal population active at the stimulation frequency may influence the phase of the response.

Given that amplitude topography was similar across conditions, it seems likely that posterior phase acceleration reflects a higher amount of synchronicity in apical dendrite synaptic activity, whereas anterior delay could reflect activation of extended, widespread neuronal assemblies being initiated later on in each cycle. Our data cannot contribute to precise neuroanatomical localization of these effects, however. EEG data are limited regarding spatial resolution, even in cases in which the number of channels is high. The aim of our study was to study basic parameters of the 10-Hz ssVEP in the sensor space, leading to estimates of phase and amplitude for voltage gradients generated by neural mass activity. Both measures have been shown to be meaningful parameters of visual processing (Silberstein et al., 1990). As a drawback of source estimation approaches, phase information is hard to interpret in EEG source space, where the orientation of dipoles used for the inverse model must be taken into account (Hauk, Keil, Elbert, \& Müller, 2002). In order to test speculations on neuroanatomical correlates underlying the present EEG results, there is research underway that employs magnetoencephalography and measures of source coherence.

In terms of specific contents, both pleasant and neutral pictures showed enhanced ssVEP amplitude for the content rated higher on arousal - that is, erotica and persons were associated with higher amplitudes than were families and household objects, respectively. For the unpleasant category, however, higher amplitudes were consistently observed during the viewing of threat pictures, as compared with mutilation pictures, the former category being subjectively rated as less arousing than the latter. Thus, within the category of unpleasant pictures, variations on the arousal dimension alone were not related to corresponding changes of ssVEP amplitude. This finding of enhanced response for threat than for mutilation stimuli is consistent with evidence from work in which startle modulation paradigms have been used (Hamm, Cuthbert, Globisch, \& Vaitl, 1997). In particu- lar, Bradley et al. (2001) have found greater startle blink potentiation and heart rate deceleration in response to threat content, as compared with other unpleasant categories, such as mutilation, pollution, or illness. In the same study, subjective arousal ratings and skin conductance scores were highest for mutilation content. Our finding of enhanced visual processing in the presence of threat is concordant with the hypothesis of motivated attention, since threat has a more immediate relationship with defensive action than have pictures showing mutilation or disease. Hence, modulation of visual processes by arousal in the aversive system appears to be closely related to defensive activation as indexed by heart rate and blink measures.

The ssVEP differences between affectively neutral and affectively arousing pictures (i.e., pleasant and unpleasant) showed a right-hemispheric preponderance. However, the present data support the view that both hemispheres contribute to affective processing. Furthermore, the temporal and spatial patterns of activation in the structures involved in affective processing in each hemisphere may play an important role in the affective processing of external stimuli and their categorization with respect to appetitive or aversive motivational states. The present findings are consistent with previous results from high-density electrophysiological recordings of oscillatory brain activity (Müller, Keil, Gruber, \& Elbert, 1999), showing a lateral-temporal modulation of the induced gamma-band response (above $30 \mathrm{~Hz}$ ) by the same set of IAPS pictures. Together with the present results, this evidence highlights the role of affective modulation in right-temporal structures. Since ssVEPs have been shown to provide insights into visual affective processing, further research may employ the capability of ssVEPs to monitor central nervous correlates of attentional fluctuations or task-related changes of cognitive performance (Belmonte, 1998; Müller, Teder-Salejarvi, $\&$ Hillyard, 1998) for investigating the time course and topography of changes between experimentally induced affective states. The present findings are consistent with the view of an organization of affective stimulus processing in highly interconnected circuits including subcortical and sensory, as well as temporal neocortical, structures. ssVEPs may be useful for elucidating the spatial and temporal neural dynamics that underlie such widespread affective networks and also may allow the study of relationships between affective perception and behavior in an experimental context.

\section{REFERENCES}

Amaral, D. G., Price, J. L., Pitkänen, A., \& Carmichael, S. T. (1992). Anatomical organization of the primate amygdaloid complex. In J. P. Aggleton (Ed.), The amygdala: Neurobiological aspects of emotion, memory, and mental dysfunction. (pp. 1-66). New York: Wiley-Liss.

Anderson, A. K., \& Phelps, E. A. (2001). Lesions of the human amygdala impair enhanced perception of emotionally salient events. $\mathrm{Na}$ ture, 411, 305-309.

Belmonte, M. (1998). Shifts of visual spatial attention modulate a 
steady-state visual evoked potential. Cognitive Brain Research, 6 , 295-307.

BLAIR, R. C., \& KARNISKI, W. (1993). An alternative method for significance testing of waveform difference potentials. Psychophysiology, 30, 518-524.

Bradley, M. M., Codispoti, M., Sabatinelli, D., \& Lang, P. J. (2001). Emotion and motivation: II. Sex differences in picture processing. Emotion, 1, 300-319.

Bradley, M. M., Greenwald, M. K., Petry, M. C., \& Lang, P. J. (1992). Remembering pictures: Pleasure and arousal in memory. Journal of Experimental Psychology: Learning, Memory, \& Cognition, 18, 379-390.

Bradley, M. M., \& LANG, P. J. (1994). Measuring emotion: The selfassessment manikin and the semantic differential. Journal of Behavior Therapy \& Experimental Psychiatry, 25, 49-59.

Burkitt, G. R., Silberstein, R. B., Cadusch, P. J., \& Wood, A. W. (2000). Steady-state visual evoked potentials and travelling waves. Clinical Neurophysiology, 111, 246-258.

CACIOPPO, J. T., \& GARDNER, W. L. (1999). Emotion. Annual Review of Psychology, 50, 191-214.

Cuthbert, B. N., Schupp, H. T., Bradley, M. [M.], McManis, M., \& LANG, P. J. (1998). Probing affective pictures: Attended startle and tone probes. Psychophysiology, 35, 344-347.

Damasio, A. R. (1998). Emotion in the perspective of an integrated nervous system. Brain Research Reviews, 26, 83-86.

Davidson, R. J., Pizzagalli, D., Nitschke, J. B., \& Putnam, K. (2002). Depression: Perspectives from affective neuroscience. Annual Review of Psychology, 53, 545-574.

DAvis, M. (1998). Anatomic and physiologic substrates of emotion in an animal model. Journal of Clinical Neurophysiology, 15, 378-387.

Gilbert, C. D. (1998). Adult cortical dynamics. Physiological Reviews, 78, 467-485.

Greenhouse, S. W., \& Geisser, S. (1959). On methods in the analysis of profile data. Psychometrika, 24, 95-112.

Hamm, A. O., Cuthbert, B. N., Globisch, J., \& Vaitl, D. (1997). Fear and the startle reflex: Blink modulation and autonomic response patterns in animal and mutilation fearful subjects. Psychophysiology, 34, 97-107.

Hamm, A. O., Greenwald, M. K., Bradley, M. M., \& Lang, P. J. (1993). Emotional learning, hedonic change, and the startle probe. Journal of Abnormal Psychology, 102, 453-465.

Hartikainen, K. M., Ogawa, K. H., \& Knight, R. T. (2000). Transient interference of right hemispheric function due to automatic emotional processing. Neurop sychologia, 38, 1576-1580.

Hauk, O., Keil, A., Elbert, T., \& Müller, M. M. (2002). Comparison of data transformation procedures to enhance topographical accuracy in time-series analysis of the human EEG. Journal of Neuroscience Methods, 113, 111-122.

Hillyard, S. A., \& Anllo-Vento, L. (1998). Event-related brain potentials in the study of visual selective attention. Proceedings of the National Academy of Sciences, 95, 781-787.

Ito, T. A., Larsen, J. T., Smith, N. K., \& Cacioppo, J. T. (1998). Negative information weighs more heavily on the brain: The negativity bias in evaluative categorizations. Journal of Personality \& Social Psychology, 75, 887-900.

Junghöfer, M., Bradley, M. M., Elbert, T. R., \& Lan, P. J. (2001). Fleeting images: A new look at early emotion discrimination. Psychophysiology, 38, 175-178.

Junghöfer, M., Elbert, T., Tucker, D. M., \& Rockstroh, B. (2000). Statistical control of artifacts in dense array EEG/MEG studies. Psychophysiology, 37, 523-532.

KARNiSKI, W., Blair, R. C., \& SNider, A. D. (1994). An exact statistical method for comparing topographic maps, with any number of subjects and electrodes. Brain Topography, 6, 203-210.

Keil, A., Bradley, M. M., Hauk, O., Rockstroh, B., Elbert, T., \& LANG, P. J. (2002). Large-scale neural correlates of affective picture processing. Psychophysiology, 39, 641-649.

KeIl, A., Gruber, T., \& Müller, M. M. (2001). Functional correlates of macroscopic high-frequency brain activity in the human visual system. Neuroscience \& Biobehavioral Reviews, 25, 527-534.

Keil, A., Müller, M. M., Gruber, T., Wienbruch, C., Stolarova,M.,
\& Elbert, T. (2001). Effects of emotional arousal in the cerebral hemispheres: A study of oscillatory brain activity and event-related potentials. Clinical Neurophysiology, 112, 2057-2068.

Kemp, A. H., Gray, M. A., Eide, P., Silberstein, R. B., \& Nathan, P. J. (2002). Steady-state visually evoked potential topography during processing of emotional valence in healthy subjects. NeuroImage, 17, 1684-1692.

LANG, P. J. (1980). Behavioral treatment and bio-behavioral assessment: Computer applications. In J. B. Sidowski, J. H. Johnson, \& T. A. Williams (Eds.), Technology in mental health care delivery systems (pp. 119-137). Norwood, NJ: Ablex.

LANG, P. J. (1995). The emotion probe: Studies of motivation and attention (102nd Annual Convention of the American Psychological Association Distinguished Scientific Contributions Award Address, Los Angeles, 1994). American Psychologist, 50, 372-385.

Lang, P. J., Bradley, M. M., \& Cuthbert, B. N. (1990). Emotion, attention, and the startle reflex. Psychological Review, 97, 377-395.

Lang, P. J., Bradley, M. M., \& Cuthbert, B. N. (1997a). International affective picture system (IAPS): Technical manual and affective ratings. Gainesville: University of Florida, Center for Research in Psychophysiology.

Lang, P. J., Bradley, M. M., \& Cuthbert, B. N. (1997b). Motivated attention: Affect, activation, and action. In P. J. Lang, R. F. Simons, \& M. T. Balaban (Eds.), Attention and orienting: Sensory and motivational processes (pp. 97-135). Mahwah, NJ: Erlbaum.

Lang, P. J., Bradley, M. M., Fitzsimmons, J. R., Cuthbert, B. N., Scott, J. D., Moulder, B., \& NANGIA, V. (1998). Emotional arousal and activation of the visual cortex: An fMRI analysis. Psychophysiology, 35, 199-210.

MAst, J., \& Victor, J. D. (1991). Fluctuations of steady-state VEPs: Interaction of driven evoked potentials and the EEG. Electroencephalography \& Clinical Neurophysiology, 78, 389-401.

Mini, A., Palomba, D., Angrilli, A., \& Bravi, S. (1996). Emotional information processing and visual evoked brain potentials. Perceptual \& Motor Skills, 83, 143-152.

Morgan, S. T., Hansen, J. C., \& Hillyard, S. A. (1996). Selective attention to stimulus location modulates the steady-state visual evoked potential. Proceedings of the National Academy of Sciences, 93, 4770-4774.

Müller, M. M., Keil, A., Gruber, T., \& Elbert, T. (1999). Processing of affective pictures modulates right-hemispheric gamma band EEG activity. Clinical Neurophysiology, 110, 1913-1920.

Müller, M. M., Picton, T. W., Valdes-Sosa, P., Riera, J., TederSalejarvi, W. A., \& Hillyard, S. A. (1998). Effects of spatial selective attention on the steady-state visual evoked potential in the 20-28 Hz range. Cognitive Brain Research, 6, 249-261.

Müller, M. M., Teder, W., \& Hillyard, S. A. (1997). Magnetoencephalographic recording of steady-state visual evoked cortical activity. Brain Topography, 9, 163-168.

Müller, M. M., Teder-Salejarvi, W., \& Hillyard, S. A. (1998). The time course of cortical facilitation during cued shifts of spatial attention. Nature Neuroscience, 1, 631-634.

Öhman, A., Fly KT, A., \& Esteves, F. (2001). Emotion drives attention: Detecting the snake in the grass. Journal of Experimental Psychology: General, 130, 466-478.

Palomba, D., Angrilli, A., \& Mini, A. (1997). Visual evoked potentials, heart rate responses and memory to emotional pictorial stimuli. International Journal of Psychophysiology, 27, 55-67.

Picton, T. W., Vajsar, J., Rodriguez, R., \& Campbell, K. B. (1987). Reliability estimates for steady-state evoked potentials. Electroencephalography \& Clinical Neurophysiology, 68, 119-131.

Plourde, G., \& Picton, T. W. (1990). Human auditory steady-state response during general anesthesia. Anesthesia \& Analgesia, 71, 460468.

REGAN, D. (1989). Human brain electrophysiology: Evoked potentials and evoked magnetic fields in science and medicine. New York: Elsevier. Schupp, H. T., Cuthbert, B. N., Bradley, M. M., Caciopro, J. T., Ito, T., \& LANG, P. J. (2000). Affective picture processing: The late positive potential is modulated by motivational relevance. Psychophysiology, 37, 257-261

Schupp, H. T., Junghöfer, M., WeIke, A. I., \& Hamm, A. O. (in press). 
Emotional facilitation of sensory processing in the visual cortex. Psychological Science.

Silberstein, R. B., Ciorciari, J., \& Pipingas, A. (1995). Steady-state visually evoked potential topography during the Wisconsin card sorting test. Electroencephalography \& Clinical Neurophysiology, 96, 24-35.

Silberstein, R. B., Harris, P. G., Nield, G. A., \& Pipingas, A. (2000). Frontal steady-state potential changes predict long-term recognition memory performance. International Journal of Psychophysiology, 39, 79-85.

Silberstein, R. B., Nunez, P. L., Pipingas, A., Harris, P., \& Danieli, F. (2001). Steady state visually evoked potential (SSVEP) topography in a graded working memory task. International Journal of Psychophysiology, 42, 219-232.

Silberstein, R. B., Schier, M. A., Pipingas, A., Ciorciari, J., Wood, S. R., \& Simpson, D. G. (1990). Steady-state visually evoked potential topography associated with a visual vigilance task. Brain Topography, 3, 337-347.
VICTOR, J. D., \& MAST, J. (1991). A new statistic for steady-state evoked potentials. Electroencephalography\& Clinical Neurophysiology, 78, 378-388.

\section{NOTE}

1. The numbers of the IAPS pictures used were as follows: pleasant, 2050, 2070, 2080, 2160, 2165, 2170, 2311, 2340, 2341, 2360, 4650, 4651, 4652, 4658, 4659, 4660, 4664, 4670, 4680, and 4690; neutral, $2190,2200,2210,2230,2381,2440,2480,2570,2850,7002,7009$, $7010,7020,7030,7040,7080,7175,7233,7235$, and 9070; unpleasant, 1050, 1120, 1201, 1300, 1930, 3000, 3010, 3050, 3060, 3071, 3080, $3102,3110,3130,3530,6260,6350,6510,6540$, and 9405 .

(Manuscript received December 10, 2002; revision accepted for publication June 11, 2003.) 